\title{
Ventricular fibrillation following high potency intravenous vitamin injection
}

\author{
R. H. FALK* \\ B.Sc., M.R.C.P.
}

\author{
Dinah E. Protheroe \\ M.A., M.B., B.Chir., M.R.C.P.
}

Brook General Hospital, London SE18

\begin{abstract}
Summary
A 67-year-old woman developed ventricular fibrillation following Parenterovite injection. Subsequent postmortem revealed normal coronary arteries. It is postulated that the high potency vitamin injection caused the arrhythmia.
\end{abstract}

\section{Introduction}

Parenterovite is a widely used proprietary parenteral vitamin preparation containing vitamins $B_{1}, B_{2}, B_{6}, C$ and nicotinamide in dextrose. Despite widespread use both intramuscularly and intravenously, side effects are rare and are usually limited to mild facial flushing due to the nicotinamide.

A case is reported of ventricular fibrillation following an intravenous injection of this preparation.

\section{Case report}

A 67-year-old woman was admitted for investigation of weight loss and progressive difficulty in walking.

Diabetes mellitus was diagnosed 6 months before admission for which she was receiving chlorpropamide $100 \mathrm{mg}$ daily. Apart from one episode of 'colitis' 25 years previously she had been well until the present illness. There was no history of allergy.

Examination revealed signs consistent with a right sided cerebello-pontine angle lesion. She was mildly hypertensive, BP $170 / 110 \mathrm{mmHg}$.

The diagnosis was confirmed by investigations, including an EMI scan.

Whilst in hospital she developed acute bloody diarrhoea which persisted for several days. Sigmoidoscopy showed friable mucosa and a rectal biopsy was consistent with ulcerative colitis, as was a barium enema.

She was started on intravenous fluids with $64 \mathrm{mg}$ i.v. prednisolone, and potassium supplements daily.

\footnotetext{
* Present address: Department of Nutrition, Harvard School of Public Health, 665 Huntington Avenue, Boston, Massachusetts 02115, U.S.A.
}

The following day the first bolus injection of a course of intravenous high potency Parenterovite was given. Immediately following this she collapsed and became pulseless.

ECG monitoring revealed ventricular fibrillation from which she made an immediate recovery with d.c. shock. Estimation of blood urea, sodium, potassium, calcium and magnesium at the time of the arrest were all normal. ECG showed minor non-specific wave changes only.

Her diarrhoea settled and one month later a large right-sided acoustic neuroma was partially removed. Unfortunately, after initial recovery, she rapidly deteriorated with signs of a brain stem haemorrhage and died.

Post-mortem confirmed the neurological findings. The heart weighed $330 \mathrm{~g}$ and showed slight left ventricular hypertrophy. The coronary arteries were free of atheroma.

\section{Comment}

Of the constituents of Parenterovite given individually, only thiamine has been reported as causing death from anaphylactic shock (Martindale, 1972; Kawasaki, Asano and Makita, 1963). This is very uncommon and the combination of thiamine with other parenteral vitamins has been said to make the risk of anaphylaxis negligible (Pollitt, 1968).

The patient described developed none of the classical manifestations of anaphylaxis although these may have been modified by the steroid administration.

Although neural mechanisms may play a part in the pathogenesis of arrhythmias in ischaemic heart disease (Lown and Verrier, 1976), there is little evidence that chronically raised intracranial pressure increases ventricular irritability, particularly in the non-ischaemic myocardium.

It is, therefore, likely that this patient's cardiac arrest was caused by the intravenous Parenterovite, possibly by the thiamine component.

Although an extremely rare complication of this drug, it is wise to have access to facilities for 
resuscitation should an intravenous bolus be given. Intramuscular preparations are available and are probably safer in out-patient practice.

\section{Acknowledgments}

Our thanks to Dr T. Fowler, consultant neurologist, for permission to publish this case.

\section{References}

Kawasaki, T., Asano, T. \& Makita, S. (1963) A case of anaphylactic shock due to intravenous thiamine hydrochloride. Japanese Journal of Medicine, 2, 168.

Lown, B. \& Verrier, R.L. (1976) Neural activity and ventricular fibrillation. New England Journal of Medicine, 294, 1165.

Martindale (1972) The Extra Pharmacopoeia. 26th edn, p. 1977. The Pharmaceutical Press, London.

Pollitt, N.J. (1968) Large intravenous dose of thiamine. Journal of the American Medical Association, 203, 153. 